\title{
Point island dynamics under fixed rate deposition
}

\author{
D. Allen ${ }^{\mathrm{a}}$, M. Grinfeld ${ }^{\mathrm{a}, *}$, R. Sasportes ${ }^{\mathrm{b}, \mathrm{c}, 1}$ \\ ${ }^{a}$ Department of Mathematics and Statistics, University of Strathclyde, Livingston Tower, \\ 26 Richmond Street, Glasgow G1 1 XH, UK \\ ${ }^{b}$ Departamento de Cièncias e Tecnologia, Universidade Aberta, Lisboa, Portugal \\ ${ }^{c}$ Centro de Análise Matemática, Geometria e Sistemas Dinâmicas, Instituto Superior \\ Técnico, Universidade de Lisboa, Lisboa, Portugal
}

\begin{abstract}
In this paper we consider the dynamics of point islands during submonolayer deposition, in which the fragmentation of subcritical size islands is allowed. To understand asymptotics of solutions, we use methods of centre manifold theory, and for globalisation, we employ results from the theories of compartmental systems and of asymptotically autonomous dynamical systems. We also compare our results with those obtained by making the quasi-steady state assumption. Keywords: submonolayer deposition, centre manifold theory, compartmental systems, asymptotically autonomous dynamical systems
\end{abstract}

2010 MSC: 34A35, 34E05, 37C19, 82D30

\section{Introduction}

Submonolayer deposition, a process in which atoms or molecules are deposited onto a substrate, diffuse and form islands, is a foundational technology in the creation of smart and nanomaterials [12]. A mathematical theory of submono-

\footnotetext{
This paper is dedicated to the memory of Jack Carr

* Corresponding author

Email addresses: damien.allen@strath.ac.uk (D. Allen), m.grinfeld@strath.ac.uk (M. Grinfeld), rafael.sasportes@uab.pt (R. Sasportes)

${ }^{1}$ Partially funded by FCT/Portugal through project RD0447/CAMGSD/2015.
} 
layer deposition that describes spatial distribution and the size statistics of the islands is an important goal of research. At present there are many competing models to describe the spatial distribution of islands; see, for example [15], and the work that paper has led to.

Size distribution of islands is usually tackled by models that disregard the spatial structure, and deal only with coagulation and fragmentation of clusters composed of adatoms deposited onto a surface. Such models lead to infinite systems of ordinary differential equations (ODEs) for the various species, these are known as rate equations; see e.g. $[9,7]$.

If furthermore one assumes that the structure of these clusters is also disregarded, one deals with point islands, and then it makes sense to assume that coagulation and fragmentation rates are not size-dependent. Studies of this type of rate equations have been initiated by da Costa et al. [4]; see also [6, 5], all of which are relevant to the present work.

As in [6] we further assume that there exists a critical island size $i$ such that islands (adatom clusters) of size $j \geq n:=i+1$ are immobile and can only grow by attachment of single adatoms.

There is a number of possibilities how to model islands of size $1<j \leq i$. The one considered in [6] is that clusters of size $1<j \leq i$ simply do not arise. There is one other physically relevant possibility, i.e. that clusters of every size $1<j \leq i$ are allowed to fragment (at some rate independent of the cluster size, which is consistent with the point-island assumption). This possibility has been considered formally in $[2,13]$. In this paper we consider this mechanism, basically using centre manifold techniques [3] and globalising the results.

In [4] and in [6] as well, it was possible by a change of variables, to decouple the infinite system of ODEs in a way that reduced its analysis to an analysis of a two-dimensional system. In our case, the reduction is to $n=i+1$ equations, and the remarkable property of these equations is that the complexity of the 
calculations is independent of $n$. Furthermore, it appears that computations can be significantly simplified by making a sweeping assumption that all the clusters of size $1<j \leq i$ are at a quasi-steady state (the quasi-steady state assumption, QSSA). We show that making this assumption results in the same leading term behaviour as the centre manifold computation and emphasise the differences between the two approaches.

The plan of the paper is as follows. In section 2 we introduce the governing equations, perform the finite dimensional reduction, and formulate an equivalence theorem between the reduced $n$-dimensional system of equations and the original infinite-dimensional one. In section 3 we perform the centre manifold analysis; the monomer asymptotics for large times are computed in section 5 and are used to discuss the consequences for the asymptotic behaviour of islands of all sizes. In section 4 we globalise these results. In section 6 we compare our results to those obtained by making the QSSA, and finally in section 7 we relate our results to those of [2] and [13] and conclude.

\section{Governing Equations}

We consider a system containing clusters of any number $j \geq 1$ or monomers; We assume that the following reactions occur:

$$
j-\text { mer }+ \text { monomer } \underset{\beta}{\stackrel{1}{\rightleftarrows}}(j+1)-\text { mer }
$$

for $1 \leq j<i$ and

$$
j-\text { mer }+ \text { monomer } \stackrel{1}{\rightarrow}(j+1)-\text { mer. }
$$

if $j \geq i$. In other words, we allow clusters of size less than $i+1$ to fragment at a rate $\beta>0$.

If we set $\tilde{\alpha}$ to be the deposition rate, denote by $C_{j}(t)$ the concentration of $j$ mers. and use primes for differentiation with respect to time $t$, the laws of mass kinetics give us the following infinite system of ODEs: 


$$
\begin{aligned}
C_{1}^{\prime} & =\tilde{\alpha}-2 C_{1}^{2}+2 \beta C_{2}-C_{1} \sum_{k=2}^{\infty} C_{k}+\beta \sum_{k=3}^{i} C_{k}, \\
C_{j}^{\prime} & =C_{1} C_{j-1}-C_{1} C_{j}-\beta C_{j}+\beta C_{j+1}, \quad 1<j<i, \\
C_{j}^{\prime} & =C_{1} C_{j-1}-C_{1} C_{j}-\beta C_{j}, \quad j=i, \\
C_{j}^{\prime} & =C_{1} C_{j-1}-C_{1} C_{j}, \quad j>i .
\end{aligned}
$$

It makes sense to scale the variables and the deposition rate to remove $\beta$ from the equations. Thus scaling $t \rightarrow T:=\beta t$, retaining primes for differentiation with respect to the new times scale, setting $C_{j}(t)=\beta c_{j}(T)$ and $\alpha=\tilde{\alpha} / \beta^{2}$, we obtain the system

$$
\begin{aligned}
& c_{1}^{\prime}=\alpha-2 c_{1}^{2}+2 c_{2}-c_{1} \sum_{k=2}^{\infty} c_{k}+\sum_{k=3}^{i} c_{k}, \\
& c_{j}^{\prime}=c_{1} c_{j-1}-c_{1} c_{j}-c_{j}+c_{j+1}, \quad 1<j<i, \\
& c_{j}^{\prime}=c_{1} c_{j-1}-c_{1} c_{j}-c_{j}, \quad j=i, \\
& c_{j}^{\prime}=c_{1} c_{j-1}-c_{1} c_{j}, \quad j>i .
\end{aligned}
$$

We next compactify the equations by setting $z=\sum_{k=2}^{\infty} c_{k}$ and $v=\alpha-c_{1} z$. Then $v$ satisfies the equation

$$
\begin{aligned}
v^{\prime}= & -\frac{1}{c_{1}}\left[c_{1}^{4}-c_{1}^{2} c_{2}+\alpha v-v^{2}-2 \alpha c_{1}^{2}+2 c_{1}^{2} v+2 \alpha c_{2}\right. \\
& \left.-2 c_{2} v+\alpha \sum_{k=3}^{i} c_{k}-v \sum_{k=3}^{i} c_{k}\right] .
\end{aligned}
$$

Then the equation for $c_{1}$ becomes

$$
c_{1}^{\prime}=v-2 c_{1}^{2}+2 c_{2}+\sum_{k=3}^{i} c_{k} .
$$

Theorem 1. If $\sum_{k=1}^{\infty} c_{k}(0)<\infty$, a solution of (4), (2) for $j>1$ and (3) is also a solution of (2) (for all $j \geq 1$ ). 
The argument of the proof is the same as in [4, Theorem 1].

Note that this means that the equations (2) for $1<j \leq i$, together with (3) and (4) can be decoupled from the rest.

We now change time from $T$ to $\tau=\int_{0}^{T} \frac{1}{c_{1}(s)} d s$. Letting dots represent the derivatives with respect to $\tau$, the $i+1$ ODEs for $v$ and $c_{j}, 1 \leq j \leq i$ become

$$
\begin{aligned}
& \dot{c}_{1}=c_{1}\left(v-2 c_{1}^{2}+2 c_{2}+\sum_{k=3}^{i} c_{k}\right), \\
& \dot{c}_{j}=c_{1}\left(c_{1} c_{j-1}-c_{1} c_{j}-c_{j}+c_{j+1}\right), \quad 1<j<i, \\
& \dot{c}_{i}=c_{1}\left(c_{1} c_{i-1}-c_{1} c_{i}-c_{i}\right),
\end{aligned}
$$

and

$\dot{v}=-\left[c_{1}^{4}-c_{1}^{2} c_{2}+\alpha v-v^{2}-2 \alpha c_{1}^{2}+2 c_{1}^{2} v+2 \alpha c_{2}-2 c_{2} v+\alpha \sum_{k=3}^{i} c_{k}-v \sum_{k=3}^{i} c_{k}\right]$.

Note that $0 \in \mathbb{R}^{i+1}$ is a rest point of the system of equations (5)-(6). The object of interest is to establish stability properties of this rest point and the way in which it is approached.

It is useful to make another change of variable. We set

$$
w=v+2 c_{2}+\sum_{k=3}^{i} c_{k}
$$

In the $\left(c_{1}, c_{2}, \ldots, c_{i}, w\right)$ variables $(4)$ becomes conveniently

$$
\dot{c}_{1}=c_{1}\left(w-2 c_{1}^{2}\right)
$$

the equations for $c_{2}, \cdots, c_{i}$ remain as before in (5) and obviously we have

$$
\begin{aligned}
\dot{w} & =\dot{v}+2 \dot{c}_{2}+\sum_{k=3}^{i} \dot{c}_{k}=-\alpha w+2 \alpha c_{1}^{2}-2 c_{1} c_{2}+2 c_{1}^{3}+c_{1} c_{3}+4 c_{1}^{2} c_{2}-2 c_{1}^{2} w \\
& -2 c_{2} w-c_{1}^{2} c_{i}-w \sum_{k=3}^{i} c_{k}+2 c_{1}^{2} \sum_{k=3}^{i} c_{k}-c_{1}^{4}+w^{2} .
\end{aligned}
$$




\section{Centre Manifold Analysis}

Now we appeal to centre manifold theory [3], according to which in a neighbourhood of the origin in $\mathbb{R}^{i+1}$, equations (7), (5) for $1<j \leq i$ and (8) admit an $i$-dimensional centre manifold, $w=h\left(c_{1}, c_{2}, \cdots, c_{i}\right)$.

On this centre manifold, the flow is given by

$$
\begin{aligned}
& \dot{c}_{1}=c_{1}\left(h\left(c_{1}, c_{2}, \cdots, c_{i}\right)-2 c_{1}^{2}\right), \\
& \dot{c}_{j}=c_{1}\left(c_{1} c_{j-1}-c_{1} c_{j}-c_{j}+c_{j+1}\right), \quad 1<j<i, \\
& \dot{c}_{i}=c_{1}\left(c_{1} c_{i-1}-c_{1} c_{i}-c_{i}\right) .
\end{aligned}
$$

But we can reparameterise time by going back to the $T$ variable to obtain on the centre manifold $w=h(\cdot)$ the equations

$$
\begin{aligned}
c_{1}^{\prime} & =h\left(c_{1}, c_{2}, \cdots, c_{i}\right)-2 c_{1}^{2}, \\
c_{j}^{\prime} & =c_{1} c_{j-1}-c_{1} c_{j}-c_{j}+c_{j+1}, \quad 1<j<i, \\
c_{i}^{\prime} & =c_{1} c_{i-1}-c_{1} c_{i}-c_{i} .
\end{aligned}
$$

Since by centre manifold theory the asymptotic expansion of $h(\cdot)$ contains only quadratic terms and above, this means that inside the centre manifold $w=h(\cdot)$ there is another, one-dimensional centre manifold, i.e. a curve parameterised by $c_{1}$, with components $c_{j}=g_{j}\left(c_{1}\right), 1<j \leq i$. We will write $g_{w}\left(c_{1}\right)=$ $h\left(c_{1}, g_{2}\left(c_{1}\right), \ldots, g_{i}\left(c_{1}\right)\right)$. Furthermore, we also know by centre manifold theory that that as $c_{1} \rightarrow 0$,

$$
g_{j}\left(c_{1}\right) \sim \sum_{k=2}^{\infty} \gamma_{j, k} c_{1}^{k}
$$

where we use $\sim$ to mean "is asymptotic to as $c_{1} \rightarrow 0$ ". We also have

$$
g_{w}\left(c_{1}\right) \sim \sum_{k=2}^{\infty} \gamma_{w, k} c_{1}^{k}
$$

Hence (see [3]) the flow on the one dimensional centre manifold is given by

$$
c_{1}^{\prime}=g_{w}\left(c_{1}\right)-2 c_{1}^{2},
$$


and if the rest point at the origin of the one-dimensional ODE (11) is stable, the one-dimensional centre manifold $\left(g_{2}\left(c_{1}\right), \ldots, g_{i}\left(c_{1}\right)\right)$ attracts nearby solutions, so all (apart possibly from sets of zero $i+1$-dimensional Lebesgue measure) approach the origin along this curve.

We have

Theorem 2. $c_{1}$ asymptotically satisfies the differential equation

$$
c_{1}^{\prime} \sim \frac{1}{\alpha}\left(-c_{1}^{i+3}+c_{1}^{i+4}-c_{1}^{2 i+3}\right)+O\left(c_{1}^{2 i+4}\right) .
$$

Proof: Before we start the computation of the coefficients $\gamma_{j, k}$ and $\gamma_{w, k}$, let us indicate the flow of logic. The equations we are dealing with, (10) and (8), have a very special structure that we are going to exploit.

On the centre manifold, the equations determining $g_{j}\left(c_{1}\right)(2 \leq j \leq i)$ have the form

$$
\begin{aligned}
& \frac{d g_{2}}{d c_{1}}\left(c_{1}\right)\left(g_{w}\left(c_{1}\right)-2 c_{1}^{2}\right)=c_{1}^{2}-c_{1} g_{2}\left(c_{1}\right)+c_{1} g_{3}\left(c_{1}\right)-g_{2}\left(c_{1}\right)+g_{3}\left(c_{1}\right) \\
& \frac{d g_{j}}{d c_{1}}\left(c_{1}\right)\left(g_{w}\left(c_{1}\right)-2 c_{1}^{2}\right)=c_{1} g_{j-1}\left(c_{1}\right)-c_{1} g_{j}\left(c_{1}\right)-g_{j}\left(c_{1}\right)+g_{j+1}\left(c_{1}\right), 2<j<i \\
& \frac{d g_{i}}{d c_{1}}\left(c_{1}\right)\left(g_{w}\left(c_{1}\right)-2 c_{1}^{2}\right)=c_{1} g_{i-1}\left(c_{1}\right)-c_{1} g_{i}\left(c_{1}\right)-g_{i}\left(c_{1}\right),
\end{aligned}
$$

to which we, denoting the right-hand side of (8) by $F\left(c_{1}, c_{2}, \ldots, c_{i}, w\right)$, add the equation

$$
\frac{d g_{w}}{d c_{1}}\left(c_{1}\right)\left(g_{w}\left(c_{1}\right)-2 c_{1}^{2}\right)=F\left(c_{1}, g_{2}\left(c_{1}\right), \ldots, g_{i}\left(c_{1}\right), g_{w}\left(c_{1}\right)\right) .
$$

By inspection, one can immediately determine $\gamma_{w, 2}$, then consecutively $\gamma_{i, 2}$, $\gamma_{i-1,2}$ and all the way to $\gamma_{2,2}$. Once this is done, we can find $\gamma_{w, 3}$ and proceed in that way to find as many terms of the expansion as we wish. See [1] for details of the form of later terms of the expansion.

For example, we have

Proposition 3. For all $j, 2 \leq j \leq i, g_{j}\left(c_{1}\right)=O\left(c_{1}^{j}\right)$ and $\gamma_{j, j}=1$. 
The final result is the following representation:

Proposition 4. The functions $g_{j}\left(c_{1}\right), 2 \leq j<i, g_{i}\left(c_{1}\right)$, and $g_{w}\left(c_{1}\right)$ have the following asymptotic expansions as $c_{1} \rightarrow 0$ :

$$
g_{j}\left(c_{1}\right) \sim c_{1}^{j}-c_{1}^{i+1}+c_{1}^{i+j}+O\left(c_{1}^{i+j+2}\right), \quad g_{i}\left(c_{1}\right) \sim c_{1}^{i}-c_{1}^{i+1}+c_{1}^{2 i}+O\left(c_{1}^{2 i+1}\right),
$$

and

$$
g_{w}\left(c_{1}\right) \sim 2 c_{1}^{2}+\frac{1}{\alpha}\left(-c_{1}^{i+3}+c_{1}^{i+4}-c_{1}^{2 i+3}\right)+O\left(c_{1}^{2 i+4}\right) .
$$

Given this Proposition, the result of Theorem 2 follows immediately.

Note that beyond terms of $O\left(c_{1}^{i+j}\right)$ the interplay among $g_{j}\left(c_{1}\right), 1<j \leq i$, and $g_{w}\left(c_{1}\right)$ becomes complex, and that the later coefficients of these functions depend on $\alpha$. Computations using MAPLE [1] indicate that the radius of convergence of the various expansions is 0 for all $\alpha>0$.

\section{Globalisation}

In this section we consider the global dynamics of equations satisfied by $c_{j}(T)$, $1 \leq j \leq i$, and $v(T)=\alpha-c_{1}(T) \sum_{k=2}^{\infty} c_{k}(T)$, and establish that all solutions of these equations with non-negative initial data approach the origin. This in turn establishes that the flow on the centre manifold, as given by Theorem 2 , describes the asymptotics of every non-negative solution of this system of equations.

For that purpose, it is more convenient to rewrite these equations as follows:

$$
\begin{aligned}
c_{1}^{\prime} & =\alpha-2 c_{1}^{2}+2 c_{2}-c_{1} \sum_{k=2}^{i} c_{k}+\sum_{k=3}^{i} c_{k}-c_{1} y, \\
c_{j}^{\prime} & =c_{1} c_{j-1}-c_{1} c_{j}-c_{j}+c_{j+1}, \quad 1<j<i, \\
c_{i}^{\prime} & =c_{1} c_{i-1}-c_{1} c_{i}-c_{i}, \\
y^{\prime} & =c_{1} c_{i},
\end{aligned}
$$


where we have put $y(T)=\sum_{k=i+1}^{\infty} c_{k}(T)$.

We first consider a system without outflows through higher clusters, i.e.

$$
\begin{aligned}
& c_{1}^{\prime}=\alpha-2 c_{1}^{2}+2 c_{2}-c_{1} \sum_{k=2}^{i} c_{k}+\sum_{k=3}^{i} c_{k}, \\
& c_{j}^{\prime}=c_{1} c_{j-1}-c_{1} c_{j}-c_{j}+c_{j+1}, \quad 1<j<i, \\
& c_{i}^{\prime}=c_{1} c_{i-1}-c_{1} c_{i}-c_{i} .
\end{aligned}
$$

Let us show that the system (13) is a compartmental system in the sense of Jacquez and Simon []. To that end, we introduce some notation.

Let $I_{1}=\alpha$ and let $I_{j}=0$ for all $2 \leq j \leq i$. Now put

$$
\begin{aligned}
& F_{j 1}=c_{1} c_{j-1}, \quad j=2, \ldots, i \\
& F_{12}=2 c_{2} \text { and } F_{1 j}=c_{j} j=3, \ldots, i .
\end{aligned}
$$

For $k=j-1,2 \leq k \leq i-1$ put $F_{k j}=c_{j}, F_{j k}=c_{1} c_{k}$ and for $k=j+1$, $2 \leq j \leq i-1$, put $F_{k j}=c_{j}$. Finally, let $F_{0 k}=0$ if $k \neq 1, i$ and $F_{0 i}=F_{01}=c_{1} c_{i}$, the only outflows from the system.

Then clearly for each $j=1, \ldots i$ we can write

$$
c_{j}^{\prime}=\sum_{k \neq j}^{i}-F_{k j}+F_{j k}+I_{j}-F_{0 j},
$$

with all the $F$ s and $I$ s positive, which shows that (13) is a compartmental system.

Also note that

$$
\frac{\partial F_{j k}}{\partial c_{m}} \geq 0 \text { for all } 1 \leq j, k, m \leq i, \quad j \neq k .
$$

Then we can use the theorem of Maeda, Kodama and Ohta [11]; see also part (i) of Theorem 9 of [10]: 
Theorem 5 ([11]). Given a compartmental system (14) with time-independent inputs $I_{j}$ that satisfies the monotonicity condition (15), every non-negative solution of the system is bounded iff the system has a positive rest point.

Since it is not hard to compute that the system (13) admits the unique positive equilibrium

$$
\left(c_{1}, c_{2}, \ldots, c_{i}\right)=\left(\alpha^{\frac{1}{i+1}}, \alpha^{\frac{2}{i+1}}, \ldots, \alpha^{\frac{i}{i+1}}\right),
$$

we conclude using Theorem ([11]) that all non-negative solutions of (13) are bounded.

Now consider the first $i$ equations of the system (12). Since the system (12) preserves non-negativity, and $y(T)$ is a positive function, by comparison with solutions of $(13)$ it follows that the $\left(c_{1}, \ldots, c_{i}\right)$ components of non-negative solutions of (12) are bounded for any positive initial condition.

Now consider the dynamics of the last component of (12), $y(T)$. As it is monotone-increasing it can either converge to some limit $l<\infty$ or it can go to infinity.

Let us show that the first possibility cannot occur. For if it did, we could use the theorem of Thieme [17, Theorem 4.2] on behaviour as $T \rightarrow \infty$ of asymptotically autonomous systems, combined with the fact that all non-negative solutions of (12) are bounded and the uniqueness of the positive equilibrium, to conclude that the $\omega$-limit set of every orbit of (12) would be the same as that of the system

$$
\begin{aligned}
c_{1}^{\prime} & =\alpha-2 c_{1}^{2}+2 c_{2}-c_{1} \sum_{k=2}^{i} c_{k}+\sum_{k=3}^{i} c_{k}-c_{1} l \\
c_{j}^{\prime} & =c_{1} c_{j-1}-c_{1} c_{j}-c_{j}+c_{j+1}, \quad 1<j<i, \\
c_{i}^{\prime} & =c_{1} c_{i-1}-c_{1} c_{i}-c_{i} .
\end{aligned}
$$

But if $y(T) \rightarrow l$ as $T \rightarrow \infty$, we must have that either $c_{1}(T) \rightarrow 0$ or $c_{i}(T) \rightarrow 0$. 
If we suppose, for example, that $c_{i}(T) \rightarrow 0$, we see from the $c_{i}^{\prime}$ equation of (16) that then either $c_{1}(T)$ or $c_{i-1}(T)$ must go to zero. Continuing in this way, we see that all $c_{j}(T)$ must go to zero as $T \rightarrow \infty$, but the origin is not a rest point of the first $i$ equations of (16). Hence we conclude that $y(T) \rightarrow \infty$.

Furthermore, since the positive orthant of $R^{i+1}$ is invariant under the flow of (12), this must mean that $c_{1}(T) \rightarrow 0$ as $T \rightarrow \infty$.

Now, from the equations for $c_{i}, c_{i-1}, \ldots, c_{2}$ it follows consecutively that for all $2 \leq k \leq i, c_{k}(T) \rightarrow 0$ as $T \rightarrow \infty$, again using the same result of Thieme [17] for asymptotically autonomous differential equations. Applying these results to the $c_{1}$ equation in (12) we finally conclude that $c_{1}(T) y(T) \rightarrow \alpha$ as $T \rightarrow \infty$, which is equivalent to saying that $v(T) \rightarrow 0$ as $T \rightarrow \infty$.

We collect these results as a theorem:

Theorem 6. As $T \rightarrow \infty$, for all non-negative initial data, $c_{k}(T) \rightarrow 0,1 \leq k \leq$ $i$, and

$$
c_{1}(T) \sum_{k=2}^{\infty} c_{k}(T) \rightarrow \alpha
$$

\section{Asymptotics of Solutions}

Armed with Theorem 2, which holds for any non-negative solution of (2) by the globalisation results of Section 4, we can discuss asymptotics of solutions of (1), using the methods of $[4,5]$, which were also used in [6]. As proofs are similar to those used in the above papers, they are omitted here; see for details [1]. Further terms in the expansions are computed using the machinery of [5]; here we just state the leading terms, denoting higher order terms by h.o.t. Going back to our original variables $C_{j}(t)$ to exhibit the complicated dependence of the results on $\beta$, we have the following statements: 
Lemma 7. The asymptotics of $C_{1}(t)$ are given by

$$
C_{1}(t) \sim\left(\frac{\tilde{\alpha} \beta^{i-1}}{(i+2) t}\right)^{\frac{1}{i+2}}+\text { h.o.t. }
$$

Note that if we set $\beta=1$ in the equation above we obtain the same result as in [6]. Already at the level of $C_{1}(t)$ one sees that the influence of the fragmentation rate $\beta$ is not intuitive.

Once we know the asymptotics of $C_{1}(t)$ from Lemma 7 , the asymptotics of $C_{j}(t)$ when $1 \leq j \leq i$ follow from Proposition 4 :

Lemma 8. The asymptotics of $c_{j}(t)$ when $1 \leq j \leq i$ are given by

$$
C_{j}(t) \sim\left(\frac{\tilde{\alpha} \beta^{\frac{i-3 j+2}{j}}}{(i+2) t}\right)^{\frac{j}{i+2}}+\text { h.o.t. }
$$

Hence we are now in a position to express the asymptotics of $C_{j}(t)$ when $j>i$ basically solving linear non-homogeneous ODEs as in [4]:

Lemma 9. The asymptotics of $C_{j}(t)$ when $j>i$ are given by

$$
C_{j}(t) \sim\left(\frac{\tilde{\alpha} \beta^{\frac{-2 i+2}{i}}}{(i+2) t}\right)^{\frac{i}{i+2}}+\text { h.o.t. }
$$

From this information we have the equivalent of Theorem 6 of [6] concerning similarity solutions of (1). To formulate it, we set

$$
\langle j\rangle=\frac{\sum_{j=1}^{\infty} j C_{j}(t)}{\sum_{j=1}^{\infty} C_{j}(t)} \sim\left(\frac{\tilde{\alpha} \beta^{i-1}}{i+2}\right)^{\frac{1}{i+2}} t^{\frac{i+1}{i+2}}+\text { h.o.t }
$$

and the function $\Psi(\eta)$ given by

$$
\Psi(\eta)= \begin{cases}(1-\eta)^{-\frac{i}{i+1}} & \text { if } \eta<1, \\ 0 & \text { otherwise. }\end{cases}
$$

With these definitions, we have 
Theorem 10. As $t \rightarrow \infty$

$$
C_{j}(t)= \begin{cases}\langle j\rangle^{-\frac{i}{i+1}} \Psi\left(\frac{(i+1) \beta^{-\frac{i+1}{i+2}}}{i+2} \frac{j}{\langle j\rangle}\right) & \text { if } \frac{(i+1) \beta^{-\frac{i+1}{i+2}}}{i+2} \frac{j}{\langle j\rangle}<1, \\ 0 & \text { otherwise. }\end{cases}
$$

\section{Quasi-Steady State Assumption}

In this section we would like to investigate whether the asymptotics of solutions obtained in Section 5 based on the centre manifold of Section 3 can be recovered more easily by combining centre manifold reasoning with a largely heuristic technique that is often used in the engineering community, the quasi-steady state approximation (QSSA; see $[8,14,16])$. As in the famous example from enzyme kinetics due to Segel and Slemrod [16], we show that QSSA correctly captures the leading term asymptotics, though of course there will be differences in higher order terms.

We restart with equations (2), but now we immediately make the QSSA assumption that $c_{j}^{\prime}=0$ for $1<j \leq i$. Now we solve these $i$ algebraic equations for $c_{j}, 1<j \leq i$, in terms of $c_{1}$. This clearly can be done consecutively, by starting with the $c_{i}$ equation and solving it in terms of $c_{1}$ and $c_{i-1}$, substituting the expression we get for $c_{i}$ into the $c_{i-1}$ equation and continuing in this way, till $c_{2}$ has been solved in terms of $c_{1}$, after which we back-substitute.

This procedure gives us that under the QSSA assumption

$$
c_{j}=\frac{\sum_{k=1}^{i-j+1} c_{1}^{k+j-1}}{\sum_{k=1}^{i} c_{1}^{k-1}}, j=2, \ldots i .
$$

Note that these are global objects, defined for all values of $c_{1}>0$. We will need their Maclaurin expansions around 0,

$$
c_{j}=c_{1}^{j}+\sum_{k=1}^{n}\left(-c_{1}^{k i+1}+c_{1}^{k i+j}\right)+O\left(c_{1}^{(n+1) i+1}\right) .
$$

Now we can go back to the equation for $\dot{w}(8)$, write $w=g_{w}\left(c_{1}\right)$, remember that by the centre manifold theorem $g_{w}$ contains terms that are at least quadratic in 
$c_{1}$, and substituting instead of $c_{j}, 1<j \leq i$, the expressions from (17), obtain that

$$
g_{w}\left(c_{1}\right) \sim 2 c_{1}^{2}+\frac{1}{\alpha} \sum_{k=1}^{n}\left(-c_{1}^{k i+3}+c_{1}^{k i+4}\right)+O\left(c_{1}^{(n+1) i+3}\right) .
$$

In the series above we can take $n$ as large as we wish. Hence under the QSSA assumption, setting $h\left(c_{1}, \ldots, c_{i}\right)=g_{w}\left(c_{1}\right)$ in the first equation of (10), the dynamics of $c_{1}$ is governed by the equation

$$
c_{1}^{\prime} \sim \frac{1}{\alpha}\left(-c_{1}^{i+3}+c_{1}^{i+4}-c_{1}^{2 i+3}+c_{1}^{2 i+4}\right)+O\left(c_{1}^{3 i+3}\right),
$$

which by inspection yields the same first three terms as the centre manifold computation of Theorem 2 for a fraction of the effort.

\section{Conclusions}

In this paper we complemented the analysis of [6] by considering a more realistic dynamics of nucleating point islands with critical island size $i$ by allowing subcritical islands of size $2 \leq j \leq i-1$ to form and fragment. The mathematics of this new system of equations is more challenging than the fundamentally 2-dimensional system considered in [6] and we had to use both centre manifold techniques and a sophisticated globalisation argument using ideas from theories of compartmental systems and of asymptotically autonomous differential equations; the globalisation methods used in this paper are in our opinion more elegant than the "brute-force" asymptotics in [6].

Our asymptotic results in section 5 are consistent with the leading term asymptotics for $c_{1}(t)$ of [13] (see our Lemma 7 ) and for $c_{j}(t)(1 \leq j \leq i)$ of [2] (see Lemma 8), as well as with the conjecture in [13] about the behaviour of $c_{j}(t)$, $j>i$ (see Lemma 9). Of course our methods are not restricted to the computation of leading terms of the asymptotics.

Acknowledgment: Part of this work was done during MG's visit to the Department of Mathematics of the University of Aveiro, whose hospitality is gratefully acknowledged. 
[1] D. Allen, Mathematical aspects of submonolayer deposition, Ph.D. thesis, University of Strathclyde, Glasgow, UK, thesis in preparation (2018).

[2] J. A. Blackman, A. Wilding, Scaling theory of island growth in thin films, Europhys. Lett. 16 (1) (1991) 115. doi:10.1209/0295-5075/16/1/020.

[3] J. Carr, Applications of Centre Manifold Theory, Springer US, 1981. doi:10.1007/978-1-4612-5929-9.

[4] F. P. da Costa, H. van Roessel, J. A. D. Wattis, Long-time behaviour and self-similarity in a coagulation equation with input of monomers, Markov Proc. and Relat. Fields 12 (2) (2006) 367-398.

[5] F. P. da Costa, J. T. Pinto, R. Sasportes, Rates of convergence to scaling profiles in a submonolayer deposition model and the preservation of memory of the initial condition, SIAM J. Math. Anal. 48 (2) (2016) 1109-1127. doi:10.1137/15m1035033.

[6] O. Costin, M. Grinfeld, K. P. O'Neill, H. Park, Long-time behaviour of point islands under fixed rate deposition, Comm. Inf. Syst. 13 (2) (2013) 183-200. doi:10.4310/cis.2013.v13.n2.a3.

[7] M. Körner, M. Einax, P. Maass, Capture numbers and island size distributions in models of submonolayer surface growth, Phys. Rev. B 86 (2012) 085403. doi:10.1103/PhysRevB.86.085403.

[8] D. A. Goussis, Quasi steady state and partial equilibrium approximations: their relation and their validity, Combustion Theory Model. 16 (5) (2012) 869-926. doi:10.1080/13647830.2012.680502.

[9] M. Einax, W. Dieterich, P. Maass, Colloquium: cluster growth on surfaces: densities, size distributions, and morphologies, Rev. Mod. Phys. 85 (2013) 921-939. doi:10.1103/RevModPhys.85.921.

[10] J. A. Jacquez, C. P. Simon, Qualitative theory of compartmental systems, SIAM Review 35 (1) (1993) 43-79. doi:10.1137/1035003. 
[11] H. Maeda, S. Kodama, Y. Ohta, Asymptotic behavior of nonlinear compartmental systems: Nonoscillation and stability, IEEE Trans. Circ. Systems 25 (6) (1978) 372-378. doi:10.1109/TCS.1978.1084490.

[12] P. Mulheran, Theory of cluster growth on surfaces, in: J. A. Blackman (Ed.), Metallic Nanoparticles, Vol. 5 of Handbook of Metal Physics, Elsevier, 2008, pp. 73 - 111. doi:10.1016/S1570-002X(08)00204-8.

[13] P. A. Mulheran, M. Basham, Kinetic phase diagram for island nucleation and growth during homoepitaxy, Phys. Rev. B 77 (2008) 075427. doi:10.1103/PhysRevB.77.075427.

[14] C. Pantea, A. Gupta, J. B. Rawlings, G. Craciun, The QSSA in Chemical Kinetics: As Taught and as Practiced, Springer Berlin Heidelberg, Berlin, Heidelberg, 2014, pp. 419-442. doi:10.1007/978-3-642-40193-0_20.

[15] A. Pimpinelli, T. L. Einstein, Capture-zone scaling in island nucleation: Universal fluctuation behavior, Phys. Rev. Lett. 99 (2007) 226102. doi:10.1103/PhysRevLett.99.226102.

[16] L. A. Segel, M. Slemrod, The quasi-steady state assumption: A case study in perturbation, SIAM Review 31 (3) (1989) 446-477. doi:10.1137/1031091.

[17] H. R. Thieme, Convergence results and a Poincaré-Bendixson trichotomy for asymptotically autonomous differential equations, J. Math. Biol. 30 (7) (1992) 755-763. doi:10.1007/BF00173267. 